\title{
Cutaneous T-Cell Acute Lymphoblastic Leukemia and the Expression Pattern of Terminal Deoxynucleotidyl Transferase Immunostaining in Mycosis Fungoides and Spongiotic Dermatitis
}

\author{
Joshua J. Clark ${ }^{a}$ Jason E. Hawkes ${ }^{a}$ b Scott R. Florella Rodney R. Miles ${ }^{\text {c, }}$ \\ David A. Wada ${ }^{a}$ d \\ a Department of Dermatology, University of Utah, Salt Lake City, UT, USA; ${ }^{b}$ Laboratory for \\ Investigative Dermatology, Rockefeller University, New York, NY, USA; ${ }^{\circ}$ Department of \\ Pathology and ARUP Laboratories, Salt Lake City, UT, USA; ${ }^{d}$ Huntsman Cancer Institute, \\ Salt Lake City, UT, USA
}

\section{Keywords}

Leukemia cutis - T-cell acute lymphoblastic leukemia - Terminal deoxynucleotidyl transferase · Immunohistochemistry

\begin{abstract}
Background/Aims: T-cell acute lymphoblastic leukemia (T-ALL) is an uncommon, aggressive malignancy that rarely presents in the skin and is generally not considered as part of the differential diagnosis by dermatologists and dermatopathologists. We describe an unusual case of T-ALL presenting with folliculocentric, erythematous papules on the face, histologically resembling mycosis fungoides (MF). Immunostaining for terminal deoxynucleotidyl transferase (TdT) was positive in tumor cells, supporting the diagnosis of cutaneous involvement by T-ALL. TdT is a nuclear enzyme expressed by immature lymphoid malignancies, but the expression pattern of this marker is not well characterized in the skin. We aimed to assess TdT staining in skin biopsies with similar-appearing lymphocytic infiltrates. Methods: We evaluated the immunostaining profile of TdT in a cohort of 23 patients, including 13 cases of MF and 10 cases of spongiotic dermatitis. Results: The lymphocytes in the MF and spongiotic dermatitis cases lacked nuclear staining for TdT. Nonspecific, granular, cytoplasmic staining was observed in a small number of background cells. Conclusions: TdT may assist dermatopathologists in discriminating malignant infiltrates of T-ALL from other conditions.




\section{Introduction}

Acute lymphoblastic leukemia (ALL) is a malignant expansion of lymphoblasts in the bone marrow, blood, and extramedullary tissues [1]. Leukemic cell infiltration of the skin (i.e., leukemia cutis) typically occurs after the diagnosis of leukemia and may precede the appearance of leukemic blasts in the peripheral blood [2]. Lymphocytic infiltrates in the skin, both malignant and benign, can have overlapping clinical and histological features, presenting a diagnostic challenge.

Terminal deoxynucleotidyl transferase (TdT) is a nuclear enzyme that polymerizes deoxynucleoside triphosphate commonly expressed in immature, pre-B and pre-T lymphoid cells [3]. TdT staining is positive in both B- and T-cell ALL (T-ALL), making it helpful in differentiating ALL from mature lymphoid malignancies [4, 5]. TdT is not commonly used by dermatopathologists, and its staining pattern in inflamed skin is poorly characterized [6-8].

We present a novel case of T-ALL-associated leukemia cutis presenting with facial nodules and histological epidermotropism with folliculotropism reminiscent of mycosis fungoides (MF). TdT immunostaining was positive in tumor cells, helping to establish the final diagnosis. This case prompted us to investigate whether TdT immunostaining reliably discriminates T-ALL from MF and other skin conditions with overlapping histological features.

\section{Index Case}

A 78-year-old Caucasian male with a history of psoriasis and psoriatic arthritis treated with etanercept presented with a 1-month history of multiple, folliculocentric, erythematous papules on the head and neck (Fig. 1). The papules originated on the right temple and were unresponsive to a month of doxycycline. He was then referred for further evaluation. A 4-mm punch biopsy from the neck was performed. Routine hematoxylin and eosin staining revealed a moderately spongiotic epidermis with monotonous, mature-appearing lymphoid cells infiltrating the epidermis and dermis (Fig. 2a) with a dense perifollicular infiltrate of atypical lymphoid cells (Fig. 2b). There were also areas of nodular and sheet-like aggregates of slightly enlarged monotonous, mononuclear lymphoid cells with scattered neutrophils. Immunohistochemical staining for CD3, CD4, CD5, CD8, CD20, CD30, and TdT was performed and demonstrated a predominantly CD4+ T-cell infiltrate that was CD5+ and largely negative for CD3 and CD8. The CD30 and CD20 stains were negative in the atypical infiltrate. TdT by immunohistochemistry subsequently revealed strong nuclear staining in $20-25 \%$ of the lymphoid cells (Fig. 2c). A peripheral blood smear revealed 49\% atypical mononuclear lymphoid cells. A bone marrow biopsy and immunophenotyping by flow cytometry demonstrated $63 \%$ lymphoid blasts of T-cell lineage that were CD34+, TdT+, CD5+, and CD3-, which was consistent with a diagnosis of T-ALL. Flow cytometry of peripheral blood displayed 58\% T lymphoblasts, of which $44 \%$ were positive for TdT. The patient was treated with modified hyper-CVAD (cyclophosphamide, vincristine, doxorubicin/Adriamycin, and dexamethasone) but unfortunately died 7 months later due to complications of fungal pneumonia and congestive heart failure.

\section{Materials and Methods}

As a result of this case, our research team sought out to evaluate the TdT staining pattern of inflammatory conditions that can mimic T-ALL, such as MF and inflammatory skin conditions with predominant lymphocytic infiltrates of the skin. This project was approved by the 
Fig. 1. Clinical photograph of index case. 78-year-old male with multiple scattered erythematous papules and nodules on the head and neck.

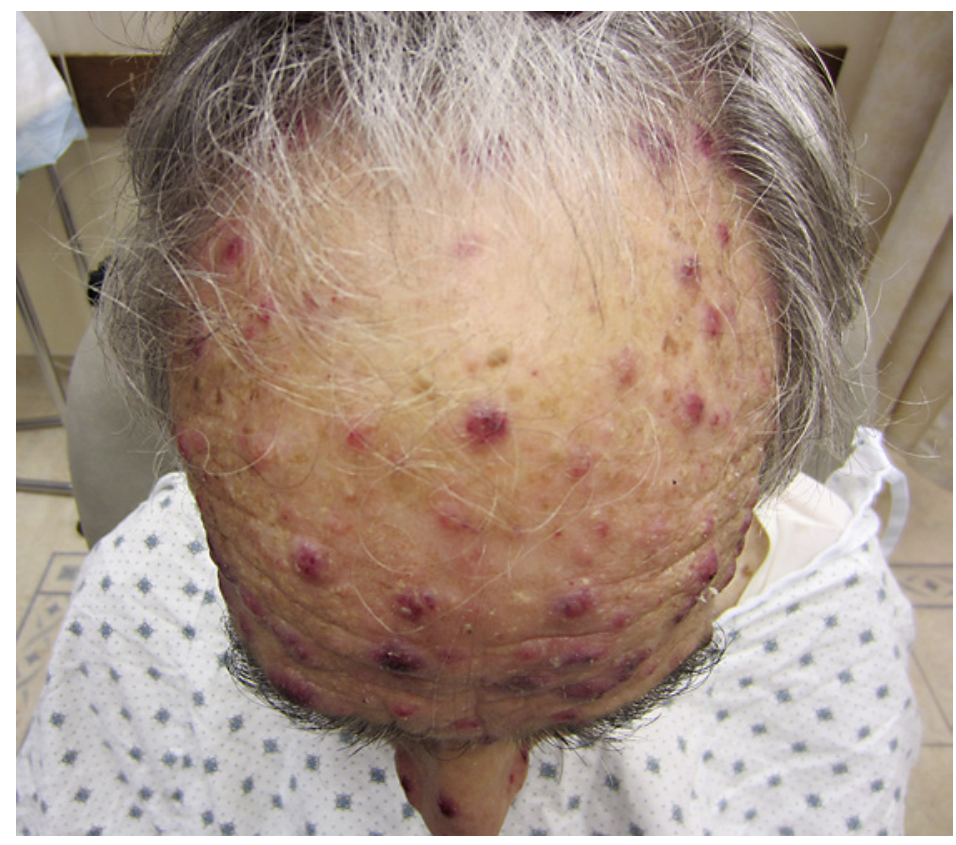

Institutional Review Board at the University of Utah (IRB_0076927). Clinical information was obtained from patient charts and pathology requisition forms. We identified 23 cases with inflammatory skin conditions for comparison with our index T-ALL case. This comparison group was composed of 13 patients with MF and 10 with spongiotic dermatitis. Prior to immunostaining, these comparison cases were selected by their overlapping histological features with our index case. Formalin-fixed slides were stained using a rabbit polyclonal anti-human TdT antibody, dilution 1:20 (SuperTech). Blinded randomized review of the 24 total slides was performed individually by select authors (J.J.C., J.E.H.), including a dermatopathologist (D.A.W.) and hematopathologist (R.R.M.), and then finalized by consensus review. TdT staining was evaluated for cell type stained, pattern of staining (i.e., nuclear, cytoplasmic, granular, or nonspecific background signal), and estimated percentage of positive-staining lymphocytes.

\section{Results}

Of the 24 TdT-stained slides (Table 1), only the index case of T-ALL demonstrated strong nuclear staining of lesional lymphocytes (Fig. 2c). Lymphocytes in the 13 cases of $\mathrm{MF}$ and 10 cases of spongiotic dermatitis were largely negative for TdT with fewer than $5 \%$ of cells having nuclear staining (online suppl. Fig. S1a; for all online suppl. material, see www.karger.com/doi/10.1159/000501581). Four cases of MF and 1 case of subacute spongiotic dermatitis with eosinophils demonstrated nuclear staining of rare, small mononuclear cells (online suppl. Fig. S1b). All cases exhibited occasional granular cytoplasmic staining of background cells (online suppl. Fig. S1c). These cells were located in the dermis and exhibited granular cytoplasmic staining that highlighted their spindle shape. Only the index case displayed strong positive nuclear staining of lymphocytes in the epidermal and dermal infiltrate. 
Clark et al.: Cutaneous T-ALL and TdT Immunostaining in MF and Spongiotic Dermatitis

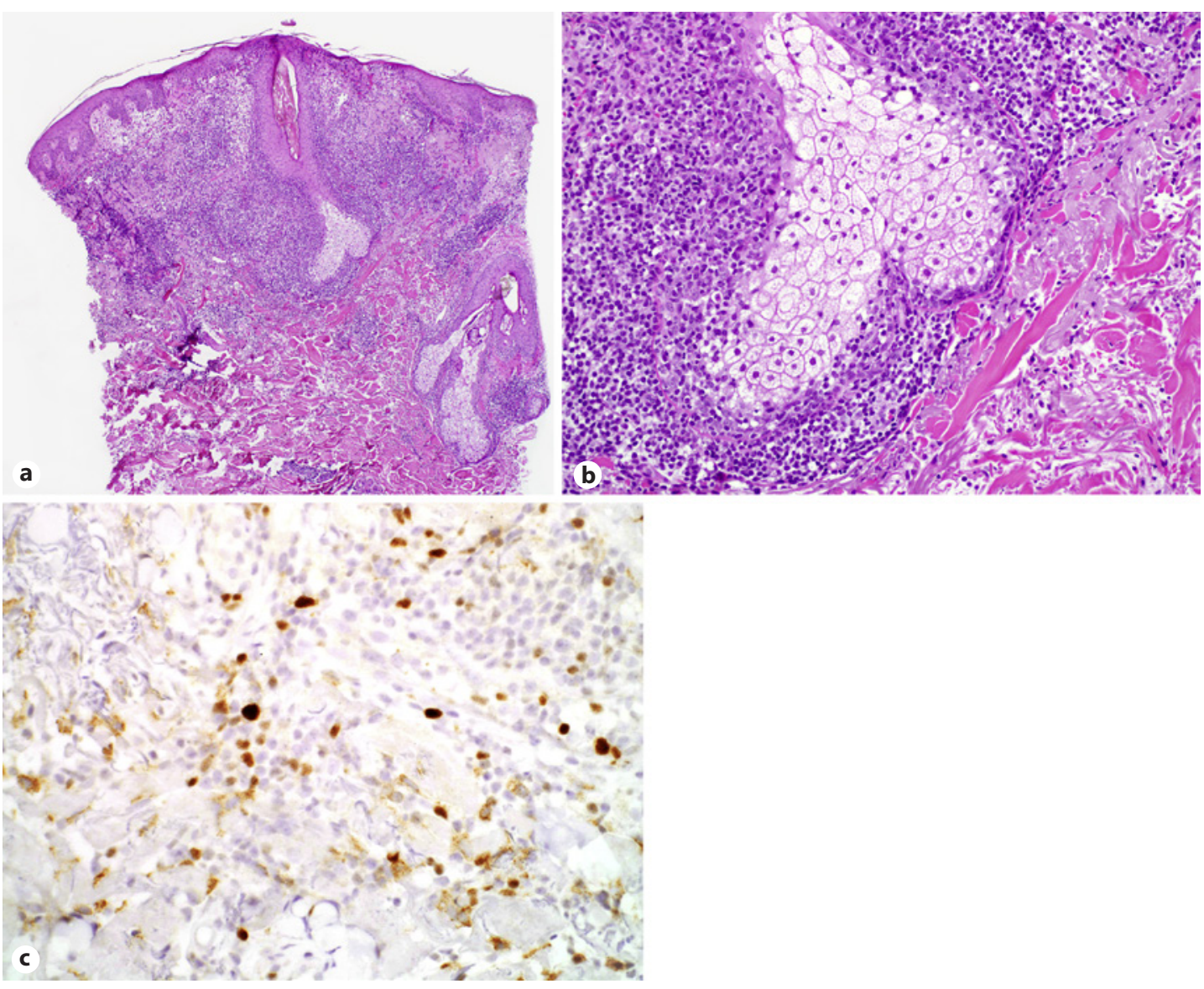

Fig. 2. Index case of cutaneous T-cell acute lymphoblastic leukemia. a Punch biopsy showing spongiotic epidermis with underlying dense dermal infiltrate of monotonous lymphoid cells. Hematoxylin-eosin. $\times 40$. b Perifollicular and follicular epithelial involvement (folliculotropism) of the atypical lymphocytic infiltrate. Hematoxylin-eosin. $\times 200$. c Strong nuclear staining of lymphoid cells and granular cytoplasmic staining in another subset of cells. TdT. $\times 400$.

Table 1. Results of TdT staining

\begin{tabular}{lccc}
\hline & $\begin{array}{l}\text { Strong nuclear } \\
\text { staining of } \\
\text { lymphocytes }\end{array}$ & $\begin{array}{l}\text { Rare small } \\
\text { mononuclear } \\
\text { cells }\end{array}$ & $\begin{array}{l}\text { Cytoplasmic } \\
\text { staining of } \\
\text { background cells }\end{array}$ \\
\hline T-ALL (index case) & $1 / 1(100 \%)$ & $1 / 1(100 \%)$ & $1 / 1(100 \%)$ \\
Mycosis fungoides & $0 / 6(0 \%)$ & $3 / 6(50 \%)$ & $6 / 6(100 \%)$ \\
Patch stage & $0 / 3(0 \%)$ & $1 / 3(33 \%)$ & $3 / 3(100 \%)$ \\
Plaque stage & $0 / 2(0 \%)$ & $0 / 2(0 \%)$ & $2 / 2(100 \%)$ \\
Interstitial & $0 / 1(0 \%)$ & $0 / 1(0 \%)$ & $1 / 1(100 \%)$ \\
Granulomatous & $0 / 1(0 \%)$ & $0 / 1(0 \%)$ & $1 / 1(100 \%)$ \\
Spongiotic dermatitis & $0 / 3(0 \%)$ & $0 / 3(0 \%)$ & $3 / 3(100 \%)$ \\
Subacute with eosinophils & $0 / 6(0 \%)$ & $1 / 6(17 \%)$ & $6 / 6(100 \%)$ \\
With mounding parakeratosis & $0 / 1(0 \%)$ & $0 / 1(0 \%)$ & $1 / 1(100 \%)$ \\
\hline Total & $1 / 24(4 \%)$ & $6 / 24(29 \%)$ & $24 / 24(100 \%)$ \\
\hline
\end{tabular}

TdT, terminal deoxynucleotidyl transferase; T-ALL, T-cell acute lymphoblastic leukemia. 
Clark et al.: Cutaneous T-ALL and TdT Immunostaining in MF and Spongiotic Dermatitis

\section{Discussion}

T-ALL is rare in the elderly population and has an incidence rate of $6.8 / 1,000,000$ persons in the USA with a median age at diagnosis of 15 years [9]. Leukemia cutis is typically seen in myeloid or mature lymphoid malignances and is estimated to occur in only 1-3\% of T- and B-cell ALL cases [10]. Most cases of T-ALL presenting with leukemia cutis have occurred in children or adolescents [10-14]. Previously described cases have demonstrated erythematous macular, papular, nodular, and plaque lesions with dense lymphoid infiltrates involving the epidermis and dermis including perivascular and periadnexal structures. Our case is an exceptional example of T-ALL presenting in the skin of an elderly adult with a histological pattern resembling MF, a far more common and less aggressive neoplasm of $\mathrm{T}$ cells [6]. To date, prior cases of T-ALL-associated leukemia cutis presenting in advanced age with epidermotropism and folliculotropism mimicking MF have not been reported.

TdT is a specialized DNA polymerase found in immature pre-B and pre-T lymphoid cells. Early investigations into the expression of this enzyme showed that TdT was one of the earliest features of cells undergoing thymus-dependent development, with loss prior to the final maturation of lymphocytes [15]. TdT staining is widely used in hematopathology, and TdT positivity has been documented in blastic natural killer cell and $\mathrm{T}$ and $\mathrm{B}$ lymphoblastic lymphoma/ leukemia [6], Merkel cell carcinoma [16], and lung carcinoma [17]. The index case of leukemia cutis was comprised of large immature lymphocytes with loose chromatin and strong, coarse nuclear staining with TdT. In contrast, the neoplastic cells in MF express a mature memory T-cell phenotype, with positivity for CD3, CD4, CD4RO, and negativity for CD8 and TdT [7, 18].

Table 1 documents the TdT staining pattern found in 23 skin biopsy specimens with inflammatory infiltrates similar to that seen in our index case. All cases showed focal granular cytoplasmic staining of background cells, which appears to be nonspecific labeling, as these cells did not have characteristic nuclear staining. We favor these to be stromal fibroblasts, histiocytes, or dendrocytes based on their size, shape, and location in the dermis. In addition, 6 of 24 cases displayed rare nuclear TdT staining of small cells presumed to be mononuclear stromal dendritic cells. While we cannot exclude the possibility that these cells are lymphocytes, we believe that this is less likely as they are easily distinguished from the TdT-positive and -negative lymphocytes in our index case by morphology, and small numbers of these cells do not seem to impact the diagnostic utility of the stain.

Limitations of our study include a small cohort and, due to the rarity of cutaneous T-ALL, an isolated index case. In addition, only $25 \%$ of T-ALL lymphocytes stained positive for TdT. This is partly explained by the presence of partially mature subpopulations of tumor cells that were negative for TdT by flow cytometry, which is known to occur in some cases [19]. Given the limited availability of tissue for this case, we were unable to assess different immunostain preparations or concentrations. Finally, the nonspecific staining requires detailed interpretation that can be challenging for pathologists lacking experience in interpreting this marker.

In summary, we present an exceptional case of T-ALL with leukemia cutis and histological features similar to MF in which TdT immunostaining was used to establish the clinical diagnosis. We then evaluated TdT staining in similar lymphocytic infiltrates that can mimic ALL and conclude that TdT may have diagnostic utility to assist dermatopathologists in discriminating ALL from other conditions when overlapping histological features are present. The presence of TdT expression in cutaneous lymphoid infiltrates should trigger the clinician to evaluate the peripheral blood and/or bone marrow to exclude the rare but aggressive malignancy of T-ALL. Further studies investigating the expression of TdT immunostaining in the skin may prove valuable to pathologists seeking to discriminate ALL from other malignant and inflammatory skin diseases. 


\section{Acknowledgments}

We would like to thank the University of Utah Dermatopathology laboratory and staff for their technical assistance and Sheryl Tripp, BS, MT (ASCP), QIHC (ASCP), along with the ARUP Institute for Clinical and Experimental Pathology for providing immunohistochemical staining.

\section{Statement of Ethics}

This project was approved by the Institutional Review Board at the University of Utah (IRB_0076927).

\section{Disclosure Statement}

The authors have no conflict of interest to declare.

\section{Funding Sources}

No funding was required for this project.

\section{Author Contributions}

All authors listed in the author byline have contributed sufficiently to the project.

\section{References}

1 Bassan R, Gatta G, Tondini C, Willemze R. Adult acute lymphoblastic leukaemia. Crit Rev Oncol Hematol. 2004; 50(3):223-61. DOI: 10.1016/j.critrevonc.2003.11.003.

2 Millot F, Robert A, Bertrand Y, Mechinaud F, Laureys G, Ferster A, et al.; The Children's Leukemia Cooperative Group of the European Organization of Research and Treatment of Cancer (EORTC). Cutaneous involvement in children with acute lymphoblastic leukemia or lymphoblastic lymphoma. Pediatrics. 1997 Jul;100(1): $60-4$.

3 McCaffrey R, Smoler DF, Baltimore D. Terminal deoxynucleotidyl transferase in a case of childhood acute lymphoblastic leukemia. Proc Natl Acad Sci USA. 1973 Feb;70(2):521-5.

4 Faber J, Kantarjian H, Roberts MW, Keating M, Freireich E, Albitar M. Terminal deoxynucleotidyl transferasenegative acute lymphoblastic leukemia. Arch Pathol Lab Med. 2000 Jan;124(1):92-7.

5 Hoffbrand AV, Janossy G. Enzyme and membrane markers in leukaemia: recent developments. J Clin Pathol. 1981 Mar;34(3):254-62.

6 Cerroni L. Lymphoproliferative lesions of the skin. J Clin Pathol. 2006 Aug;59(8):813-26.

7 Drexler HG, Menon M, Minowada J. Incidence of TdT positivity in cases of leukemia and lymphoma. Acta Haematol. 1986;75(1):12-7.

8 Mastrandrea F, Cadario G, Bedello PG, Nicotra MR, Natali PG. Expression of T-lineage early developmental markers by cells establishing atopic dermatitis skin infiltrates. J Investig Allergol Clin Immunol. 1998 Nov-Dec;8(6):359-64.

9 Howlader N, Noone AM, Krapcho M, Miller D, Bishop K, Kosary CL, et al. SEER cancer statistics review, 19752014. Natl Cancer Institute Bethesda, MD [cited 2017 April 22]. Available from: http://seer.cancer.gov/ csr/1975_2014/.

10 Jiang X, Wang W, Zhang M. Leukemia cutis: an unusual presentation of acute lymphoblastic leukemia in a child. Indian J Dermatol. 2015 Nov-Dec;60(6):636.

11 Chao SC, Lee JY, Tsao CJ. Leukemia cutis in acute lymphocytic leukemia masquerading as viral exanthem. J Dermatol. 1999 Apr;26(4):216-9. 
12 Boccara O, Blanche S, de Prost Y, Brousse N, Bodemer C, Fraitag S. Cutaneous hematologic disorders in children. Pediatr Blood Cancer. 2012 Feb;58(2):226-32.

13 Ali R, Ozan U, Ozkalemkas F, Ozcelik T, Ozkocaman V, Ozturk H, et al. Leukaemia cutis in T-cell acute lymphoblastic leukaemia. Cytopathology. 2006 Jun;17(3):158-61.

14 Najem N, Zadeh VB, Badawi M, Kumar R, Al-Otaibi S, Al-Abdulrazzaq A. Aleukemic leukemia cutis in a child preceding T-cell acute lymphoblastic leukemia. Pediatr Dermatol. 2011 Sep-Oct;28(5):535-7.

15 Silverstone AE, Cantor H, Goldstein G, Baltimore D. Terminal deoxynucleotidyl transferase is found in prothymocytes. J Exp Med. 1976 Aug;144(2):543-8.

16 Sur M, AlArdati H, Ross C, Alowami S. TdT expression in Merkel cell carcinoma: potential diagnostic pitfall with blastic hematological malignancies and expanded immunohistochemical analysis. Mod Pathol. 2007 Nov;20(11):1113-20.

17 Sidiropoulos M, Hanna W, Raphael SJ, Ghorab Z. Expression of TdT in Merkel cell carcinoma and small cell lung carcinoma. Am J Clin Pathol. 2011 Jun;135(6):831-8.

18 Willemze R, Jaffe ES, Burg G, Cerroni L, Berti E, Swerdlow SH, Ralfkiaer E, et al. WHO-EORTC classification for cutaneous lymphomas. Blood. 2005;105(10):3768-85. DOI: 10.1182/blood-2004-09-3502.An.

19 Wang H, Song C, Gurel Z, Song N, Ma J, Ouyang H, et al. Protein phosphatase 1 (PP1) and casein kinase II (CK2) regulate Ikaros-mediated repression of TdT in thymocytes and T-cell leukemia. Pediatr Blood Cancer. 2014 Dec;61(12):2230-5. 